\title{
Development and evaluation of oral Cancer quality-of-life questionnaire (QOL-OC)
}

\author{
Min Nie*, Chang Liu, Yi-Chen Pan, Chen-Xi Jiang, Bao-Ru Li, Xi-Jie Yu, Xin-Yu Wu and Shu-Ning Zheng
}

\begin{abstract}
Background: In this study scales and items for the Oral Cancer Quality-of-life Questionnaire (QOL-OC) were designed and the instrument was evaluated.

Methods: The QOL-OC was developed and modified using the international definition of quality of life (QOL) promulgated by the European Organization for Research and Treatment of Cancer (EORTC) and analysis of the precedent measuring instruments. The contents of each item were determined in the context of the specific characteristics of oral cancer. Two hundred thirteen oral cancer patients were asked to complete both the EORTC core quality of life questionnaire (EORTC QLC-C30) and the QOL-OC. Data collected was used to conduct factor analysis, test-retest reliability, internal consistency, and construct validity.

Results: Questionnaire compliance was relatively high. Fourteen of the 213 subjects accepted the same tests after 24 to $48 \mathrm{~h}$ demonstrating a high test-retest reliability for all five scales. Overall internal consistency surpasses 0.8 . The outcome of the factor analysis coincides substantially with our theoretical conception. Each item shows a higher correlation coefficient within its own scale than the others which indicates high construct validity.

Conclusions: QOL-OC demonstrates fairly good statistical reliability, validity, and feasibility. However, further tests and modification are needed to ensure its applicability to the quality-of-life assessment of Chinese oral cancer patients.
\end{abstract}

Keywords: Oral Cancer quality-of-life questionnaire, QOL-OC, Chinese oral cancer, Chinese version

\section{Background}

Oral cancer includes cancers of the oral cavity and adjacent anatomical sites. The incidence rate of oral cancer ranks sixth among systemic cancers and first among head and neck cancers [1]. Occurrence of oral cancer continues to increase and developing countries experience higher rates of morbidity and mortality from oral cancer than do developed countries [2]. Due to the specific anatomical sites and structures that characterize oral cancer, both the disease process and its treatment may greatly impair the body image and practical functions of patients in even the most essential life tasks such as breathing, speaking, swallowing, and eating [3, 4]. During the past six decades, both the mortality and 5-year survival rates among oral cancer patients basically remain unchanged $[5,6]$. For these reasons, the

\footnotetext{
* Correspondence: niemin@whu.edu.cn

The State Key Laboratory Breeding Base of Basic Science of Stomatology, Hubei Province \& Key Laboratory of Oral Biomedicine (Wuhan University), Ministry of Education, School and Hospital of Stomatology, Wuhan University, Luoyu Road 237, Wuhan 430079, Hubei, China
}

improvement of quality of life has gained the attention of the medical community [7]. Furthermore, quality of life assessments have been providing evidence that is critical to both the assessment of patients' living states and the formulation of clinical strategies [8-10].

Standardized measuring instruments are used to assess quality of life $[11,12]$. Widely used QOL instruments include the European Organization for Research and Treatment of Cancer (EORTC) QLQs [13, 14], the Functional Assessment of Cancer Therapy (FACT) scale [15], and the University of Washington Head and Neck Measure (UW-QOL) and the Functional Living IndexCancer (FLIC) [16], and so on. Neither the questionnaires mentioned above nor other generic measures like the Medical Outcomes Short Form 36 (SF-36) [17] are specifically tailored to the problems oral cancer patients experience [18, 19]. Assessment of oral cancer has been borrowing scales of head and neck cancer, such as EORTC H\&N35, FACT-H\&N, and so on. In fact, patients with oral cancer are more likely to suffer from

(c) The Author(s). 2018 Open Access This article is distributed under the terms of the Creative Commons Attribution 4.0 International License (http://creativecommons.org/licenses/by/4.0/), which permits unrestricted use, distribution, and 
more significant dental problems, more limited mouth opening, more severe swallowing, chewing, speech and saliva secretion problems than other head and neck cancers (such as laryngeal cancer, pharyngeal cancer, and so on) [20-26]. Different anatomical sites and its functions are bound to be the limitation of life questionnaire with head and neck cancer [13, 21, 27-29]. Furthermore, there is dearth of specific measures designed for use within the context of Chinese culture. Therefore, there is a need for a questionnaire designed to assess the quality of life of Chinese oral cancer patients.

\section{Methods}

\section{Development of the QOL-OC}

The questionnaire consists of a general module and a specific module. The EORTC QLQ-C30 and its core measures for cancer patients were directly adopted as the general module. The QLC-C30 contains a total of 30 questions covering the basic aspects of health-related quality of life. Its five function scales are physical functioning, role functioning, cognitive functioning, emotional functioning, and social functioning; the remainder of the questionnaire consists of symptom scales. The questionnaire is used in the treatment of patients suffering from all categories of cancer [28]. The Chinese version of the C30 enjoys relatively high reliability, validity, and feasibility as well as wide recognition in China [30].

The oral-cancer-specific module is based on the guidelines of the EORTC Quality of Life Group [31-34] as well as the definition of QOL by the World Health Organization (WHO) as an individual's "perception of their position in life in the context of culture and value system in their life and in relation to their goals, expectations, standards and concerns" [35].

Drawing on relevant papers collected from databases like PubMed and SSIC and opinions from patients and experts, we amalgamated, deleted, rephrased, and added some of the current questionnaire items. These decisions were based mainly on the current version of the EORTC $\mathrm{H} \& \mathrm{~N} 35$; the overall purpose of this work was to detect the problems in currently available measures and revise accordingly. Considering the limitations of the instrument, an open-ended question was added to supplement it.

The QOL-OC consists of 29 items among 15 scales. The first 26 of these 29 items are scored on a four-point Likert scale: meiyou (not at all), youyidian (a little), bijiaoduo (quite a bit), feichangduo (very much). Items 27 and 28 item are yes/no questions; yes responses are scored with 1 point and no responses are scored with 0 . The last item, number 29, is an open-ended question which does not contribute to the numerical score and is only used for material collection. The division of scales and the scoring procedure are shown in Table 3.

\section{Data collection}

Each subject (more than 18) signed a formal Informed Consent statement, and the entire study was approved by the Medical Ethics Committee of Wuhan University. Data collection began in June 2014 and ended in March 2015. Patients were selected from Wuhan University School of Stomatology, Wuhan University Zhongnan Hospital, Changzhou Stomatology Hospital, and Shenzhen No. 2 People's Hospital. The majority of the patients were interviewed telephonically and the rest were interviewed face-to-face. Some of the patients was interviewed again 24 to $48 \mathrm{~h}$ later solely to establish test-retest reliability. Inclusion and exclusion criteria are as follows:

Inclusion criteria:

1) Pathologically diagnosed with oral cancer;

2) 18 or older;

3) Estimated survival time longer than 3 months;

4) Aware of the diagnosis;

5) Able to understand and answer the questionnaire on their own or with the explanation of investigators;

6) Involved voluntarily.

\section{Exclusion criteria:}

1) With mental illness, disturbances in conscious mental processes, or communication barriers;

2) Refusing to be involved in this study or asking to quit during the study.

\section{Data analysis}

Statistical analysis was conducted using SPSS 19.0. Internal consistency was assessed using Cronbach's alpha, and test-retest ability was assessed using the Spearman rank-order correlation coefficient. Factor analysis (principal axis factor analysis), along with parallel analysis were conducted to judge the division of scales, and convergent and discriminant construct validity were evaluated using the Spearman rank-order correlation coefficient.

\section{Results \\ Development of questionnaire}

The EORTC-H\&N35 [13] and other commonly used head and neck measures such as FACT-H\&N [36] and UW-QOL [37] were used to develop the QOL-OC questionnaire. QOL-OC used EORTC-H\&N35 as it prototype and according to the pilot study, specific questions intimately related to the oral cavity region such as shoulder and neck function, saliva secretion were added to the instrument and less significantly related items from the sources were eliminated. This resulted in a questionnaire containing 29 questions, see Additional file 1.

Based on the information gleaned from the discussion of the panel and patients, several questions were fine- 
Table 1 Demographic information of patients included

\begin{tabular}{|c|c|c|}
\hline Characteristics & No. of patients & $\%$ \\
\hline \multicolumn{3}{|l|}{ Gender } \\
\hline Male & 139 & 65.3 \\
\hline Female & 74 & 34.7 \\
\hline \multicolumn{3}{|l|}{ Age } \\
\hline Mean (SD) & $53.84(10.48)$ & \\
\hline Range & $18-86$ & \\
\hline \multicolumn{3}{|l|}{ Marital status } \\
\hline Married & 197 & 92.5 \\
\hline Unmarried & 8 & 3.8 \\
\hline Widowed & 5 & 2.3 \\
\hline Divorced & 2 & .9 \\
\hline \multicolumn{3}{|l|}{ Education level } \\
\hline Junior high & 73 & 34.3 \\
\hline Senior high & 60 & 28.2 \\
\hline Undergraduate or higher & 41 & 19.2 \\
\hline Primary school or lower & 30 & 14.1 \\
\hline Information missed & 9 & 4.2 \\
\hline \multicolumn{3}{|l|}{ Cancer site } \\
\hline Tongue & 51 & 23.9 \\
\hline Buccal mucosa & 29 & 13.6 \\
\hline Gingiva & 25 & 11.7 \\
\hline Salivary glands & 25 & 11.7 \\
\hline Mouth floor & 23 & 10.8 \\
\hline Palate & 23 & 10.8 \\
\hline Multi-sites & 10 & 4.7 \\
\hline Lips & 10 & 4.7 \\
\hline Maxilla/Mandible & 8 & 3.8 \\
\hline Oropharynx & 5 & 2.3 \\
\hline branchial fissure & 1 & .5 \\
\hline Temple & 1 & .5 \\
\hline Maxillary sinus & 1 & .5 \\
\hline Submandibular & 1 & .5 \\
\hline \multicolumn{3}{|l|}{ Pathological type } \\
\hline SCC & 144 & 67.6 \\
\hline Mucoepidermoid carcinoma carcinoma & 12 & 5.6 \\
\hline Adenoid cystic & 11 & 5.2 \\
\hline Adenocarcinoma & 6 & 2.8 \\
\hline Pleomorphic adenoma & 4 & 1.9 \\
\hline Other & 30 & 14.6 \\
\hline Ameloblastoma & 1 & .5 \\
\hline Malignant Melanoma & 2 & .9 \\
\hline Hodgkin lymphoma & 1 & .5 \\
\hline Non-Hodgkin lymphoma & 2 & .9 \\
\hline Myoepithelial carcinoma & 3 & 1.4 \\
\hline
\end{tabular}

Table 1 Demographic information of patients included (Continued)

\begin{tabular}{lll}
\hline Characteristics & No. of patients & $\%$ \\
\hline Basal cell carcinoma & 3 & 1.4 \\
Mesenchymal sarcoma & 1 & .5 \\
Plasma cell sarcoma & 2 & .9 \\
Sarcomatoid carcinoma & 1 & .5 \\
Mesenchymal carcinoma & 2 & .9 \\
Epithelioid vascular endothelium & 1 & .5 \\
Spindle cell sarcoma & 1 & .5 \\
Fibroblastoma & 1 & .5 \\
Acinic cell carcinoma & 4 & 1.9 \\
Adenogenous low-grade malignancy & 3 & 1.4 \\
Dentinogenic ghost cell tumour & 1 & .5 \\
Verrucous carcinoma & 1 & .5 \\
Information missed & 6 & 2.8 \\
Treatment & & \\
Surgery & 200 & 93.9 \\
Surgery/radiotherapy & 8 & 3.8 \\
Radiotherapy & 2 & .9 \\
Surgery/radiotherapy/chemotherapy & 1 & .5 \\
Surgery/chemotherapy & 1 & .5 \\
Palliative treatment & 1 & \\
\hline
\end{tabular}

tuned to be more acceptable and easily understood by patients. It is worth noting that, drawing from the Chinese version of the EOTRC QLQ-C30, we used a four point scale: 'meiyou (not at all), 'youyidian (a little)', 'xiangdangduo (quite a lot)', and 'feichangduo (very much)'. However, during the discussion patients reported difficulty distinguishing between 'xiangdangduo (quite a lot)' and 'feichangduo (very much)' in Chinese. Therefore, we rephrased 'xiangdangduo' into 'bijiaoduo (relatively much)' in the actual interviews.

We analyzed data and results from the current publications and working papers concerning the appraisal of head and neck specific measuring instruments, and extracted several oral functions that were not covered in our prototype frequently complained by oral cancer patients such as shoulder and neck function [21, 38], mastication [37, 39]. We then added other oral problems that patients might confront such as oral ulcer, enjoyment of food, diet change and bleeding gingiva. Since problems with speech is much less prominent among oral cancer patients than others like laryngeal cancer patients $[13,40]$, speech item was removed and substituted by pronunciation which is more related to organs in oral cavity than throat. Sense of smell was also removed for similar reason that dysosmia occurs more on nasopharynx cancer patients [13, 41]. The two questions 
concerning sexuality were combined considering the high internal consistency $[13,19,42]$.

\section{Patient characteristics}

A total of 213 patients were included. Significantly more subjects were males and the study included patients with a broad range of cancer sites (Table 1).

\section{Compliance}

During the data collection period, a total of 282 patients were called or interviewed. Fifty-five directly refused and 10 failed to complete all the questions. Therefore, 213 effective questionnaires for each measuring instrument were obtained indicating a response rate of $75.5 \%$. Subjects spent $10.4 \mathrm{~min}$ on average completing the questionnaire.

\section{Descriptive statistics}

The patient responses to C30 described a general quality of life that was slightly low (score for global health status was $74.53 \pm 19.92$ ). Though physical functioning (90. $58 \pm 14.86)$, emotional functioning (93.19 \pm 11.21$)$, and cognitive functioning $(91.47 \pm 11.85)$ were fairly good in the context of the function scales, role functioning (88. $58 \pm 19.49)$ and social functioning $(86.31 \pm 21.14)$ lagged behind. In the context of the symptom scales, patients responses reflected prominent economic problems most often, followed by sleeping problems $(14.40 \pm 25.11)$, fatigue $(13.67 \pm 16.85)$, and pain $(10.80 \pm 16.92)$.

All QOL-OC scales are symptom scales. Notable eating $(13.42 \pm 15.11)$ and saliva problems $(22.85 \pm 23$. 06) were reported most frequently, whereas problems with pain and discomfort $(8.58 \pm 11.95)$, sexuality (6. $57 \pm 20.19)$, oral ulcers (8.45 \pm 17.48$)$, and bleeding gingival $(5.16 \pm 17.11)$ were less frequently reported (mean < 10). Weight gain $(27.70 \pm 44.86)$ occurred more than weight loss $(18.31 \pm 38.77)$. The scores of these two questionnaires can be seen in Additional files 2 and 3 .

\section{Factor analysis}

Factor analysis was performed to assess the division of scales. Parallel analysis was conducted to help decide the number of factors. Scree plot (Fig. 1) was drawn from actual eigenvalues obtained from principal axis factor analysis by varimax rotation and random eigenvalues got from parallel analysis.

According to the scree plot, 6 factors should be extracted. But considering the slightly low variances contribution (54.89\%), 7 factors (58.97\%) were selected instead [43] (Table 2). Factor 1 reflects problems of social contact. Factor 3 is mainly concerned with pain. Factor 4 is about problems with eating. Factor 5 involves problems with diet, weight change and sense of taste. Factor 7 reflects problems with saliva.

In both statistically and clinically justified sense, the final scale division was established as shown in Table 3.

\section{Reliability}

Cronbach's $\alpha$ was calculated using data collected during the first trial to confirm internal consistency. In the social contact $(\alpha=0.889)$ and diet scales $(\alpha=0.751)$ satisfying outcomes were achieved $(\alpha>0.7)$, while internal consistency was slightly lower in the pain and discomfort $(\alpha=0.677)$, eating scale $(\alpha=0.515)$, and saliva scale $(\alpha=0$. $605)$. Overall internal consistency was high $(\alpha=0.875)$.

Data acquired from both trials (with an interval of 24 to $48 \mathrm{~h}$ between trials) were used to calculate test-retest reliability; this calculation procedure consisted of comparing the scores from the two trials using correlation

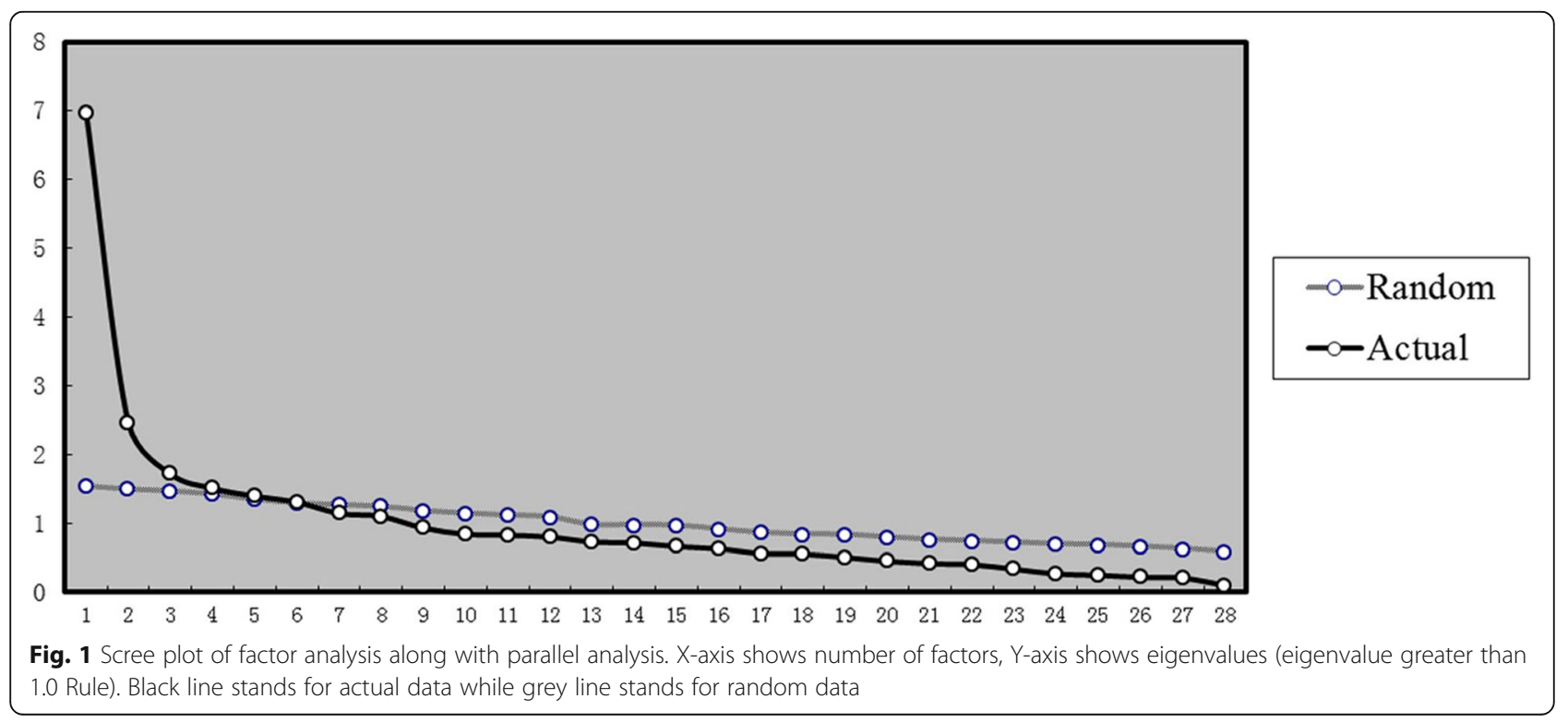


Table 2 Factor loadings of principal axis factor analysis

\begin{tabular}{|c|c|c|c|c|c|c|c|}
\hline Factors & 1 & 2 & 3 & 4 & 5 & 6 & 7 \\
\hline 1 Pain in mouth & & & .585 & & & & \\
\hline 2 Pain in jaw & & & .574 & & & & \\
\hline 3 Pain in throat & & & .343 & & .394 & & \\
\hline 4 Discomfort in mouth & & .583 & & & & & \\
\hline 5 Eating fluid & & & & .927 & & & \\
\hline 6 Eating semifluid & & & & .605 & & & \\
\hline 7 Eating solid & & .588 & & & & & \\
\hline 8 Choked when eating & & & & & & .502 & \\
\hline 9 Teeth problem & & .346 & & & & & \\
\hline 10 Difficulty opening mouth & & .529 & & & & & \\
\hline 11 Dry mouth & & & & & & & .662 \\
\hline 12 Sticky saliva & & & & & & & .533 \\
\hline 13 Sense of taste & & & & & .371 & & \\
\hline 14 Appearance & & & & & & .350 & \\
\hline 15 Difficulty chatting & .831 & & & & & & \\
\hline 16 Social contact with family & .792 & & & & & & \\
\hline $\begin{array}{l}17 \text { Social contact } \\
\text { with friends }\end{array}$ & .849 & & & & & & \\
\hline 18 Social contact in public & .819 & & & & & & \\
\hline 19 Sexuality & & & & & & .441 & \\
\hline 20 Oral ulcer & & .241 & & & & & \\
\hline 21 Trouble enjoying food & & & & & .422 & & \\
\hline 22 Diet change & & .403 & & & .394 & & \\
\hline 23 Pronunciation & .628 & & & & & & \\
\hline $\begin{array}{l}24 \text { Shoulder } \\
\text { \& neck function }\end{array}$ & & & & & & .359 & \\
\hline 25 Bleeding gingiva & & .094 & & & & & \\
\hline 26 Painkillers & & & .530 & & & & \\
\hline 27 Weight loss & & & & & .513 & & \\
\hline 28 Weight gain & & & & & -.379 & & \\
\hline
\end{tabular}

Only the maximum loadings of each item were shown in the table

coefficient $r$ (Table 3). Except for the shoulder and neck function scales, the tested scales indicated close correlation. Therefore, overall internal consistency was confirmed.

\section{Validity}

Correlation coefficients between the five scales used to establish discriminant validity are shown in Table 4 . Diet, eating, and social contact were moderately correlated $(0.478-0.551)$ while pain, saliva, and other scales demonstrated low correlation.

Correlation coefficients between items and their own scales used to establish convergent validity were calculated (Table 5). Higher correlations were observed between all items and their corresponding scales than between items and the other scales which indicated fairly good convergent validity. There was a lower correlation between the painkiller item and its scale (0.376); however, this value was still greater than the correlations between any other items and the pain and discomfort scale by horizontal or vertical standards for comparison. Finally, the eating fluid item manifested a low correlation (0.372) with the eating scale.

Correlation between the C30 and QOL-OC scales was also calculated (Table 6). QL in C30 and ET in OC $(r=0.420)$, SF in C30 and DT in OC $(r=0.450)$ were moderately correlated. There was significant correlation between PA in C30 and PD in OC $(r=0.543)$; significant correlation was also observed between SF in C30 and SC in $\mathrm{OC}(r=0.525)$.

\section{Discussion}

Head and neck cancer is a disease of the upper aerodigestive tract and is one of the most frequently diagnosed cancers worldwide. A high rate of cancers involving the head and neck are reported across the Asian region [44, 45]. To better reflect current clinical management of oral cancer within Chinese, Quality-of-life questionnaire is expected to benefit practitioners when making decisions regarding optimal treatment strategies for their patients.

The Chinese oral cancer specific quality-of-life questionnaire (QOL-OC) categorizes 29 scored questions into 15 scales, five of which are symptom scales and 10 of which are item scales. The QOL-OC also contains a single multi-choice question used for material collection only [27]. In specific clinical application, certain questions can be selectively analysed. But in clinical studies, division of scales can lower the size of data so as to reduce the work load of data analysis.

The telephonic interview was used at this stage and average completion time for the interview was $10.4 \mathrm{~min}$; this was accepted by most of the patients. But it is common for patients refused second interview, one to 2 days after the first one. Asking questions item by item guaranteed that missing data was not an issue in this study. The only missing data concerned sexuality (4.6\%), due to a lack of sexual activity among participants of advanced ages [41].

The outcome of the factor analysis coincides basically with our theory. One noteworthy result was that the pain in the throat and discomfort in the mouth items (Items 3 and 4) were placed within the pain and discomfort scale, mainly because of their relevance to clinical functions and the scale division of EORTC-H\&N35 [46]. The eating solid and being choked when eating items (Items 7 and 8) were placed within the eating scale together along with the eating fluid and eating semifluid items; this was done in order to guarantee the integrity and logic of this series of questions which concern four levels of difficulty in eating. The sense of taste which was placed within a single 
Table 3 Scoring Methods and Test-retest ability of QOL-OC

\begin{tabular}{|c|c|c|c|c|c|c|c|c|}
\hline Scale & Scales & Items & $\begin{array}{l}\text { Score } \\
\text { Range }\end{array}$ & Min & Max & Rough Score & $\begin{array}{l}\text { Standardized } \\
\text { Score }\end{array}$ & $\begin{array}{l}\text { Spearman correlation } \\
\text { coefficient }(r)\end{array}$ \\
\hline Pain and discomfort & PD & $1,2,3,4,26$ & $1 \sim 4$ & 5 & 20 & $(1+2+3+4+26) / 5$ & {$[(\mathrm{RS}-1) / 3] \times 100$} & $0.881^{* *}$ \\
\hline Eating & ET & $5,6,7,8$ & $1 \sim 4$ & 4 & 16 & $(5+6+7+8) / 4$ & {$[(\mathrm{RS}-1) / 3] \times 100$} & $0.975^{* *}$ \\
\hline Saliva & SA & 11,12 & $1 \sim 4$ & 2 & 8 & $(11+12) / 2$ & {$[(\mathrm{RS}-1) / 3] \times 100$} & $0.918^{* *}$ \\
\hline Social contact & SC & $\begin{array}{l}15,16,17,18, \\
23\end{array}$ & $1 \sim 4$ & 5 & 20 & $\begin{array}{l}(15+16+17+18+ \\
23) / 5\end{array}$ & {$[(\mathrm{RS}-1) / 3] \times 100$} & $0.939^{* *}$ \\
\hline Diet & DT & 21,22 & $1 \sim 4$ & 2 & 8 & $(21+22) / 2$ & {$[(\mathrm{RS}-1) / 3] \times 100$} & $0.923^{* *}$ \\
\hline Teeth & TE & 9 & $1 \sim 4$ & 1 & 4 & 9 & {$[(\mathrm{RS}-1) / 3] \times 100$} & $0.984^{* *}$ \\
\hline Opening mouth & OM & 10 & $1 \sim 4$ & 1 & 4 & 10 & {$[(\mathrm{RS}-1) / 3] \times 100$} & $0.938^{* *}$ \\
\hline Sense of taste & TA & 13 & $1 \sim 4$ & 1 & 4 & 13 & {$[(\mathrm{RS}-1) / 3] \times 100$} & $0.997^{* *}$ \\
\hline Appearance & AP & 14 & $1 \sim 4$ & 1 & 4 & 14 & {$[(\mathrm{RS}-1) / 3] \times 100$} & $0.817^{* *}$ \\
\hline Sexuality & SX & 19 & $1 \sim 4$ & 1 & 4 & 19 & {$[(\mathrm{RS}-1) / 3] \times 100$} & $\rho^{a}$ \\
\hline Oral ulcer & OU & 20 & $1 \sim 4$ & 1 & 4 & 20 & {$[(\mathrm{RS}-1) / 3] \times 100$} & $0.990^{* *}$ \\
\hline $\begin{array}{l}\text { Shoulder \& neck } \\
\text { function }\end{array}$ & SN & 24 & $1 \sim 4$ & 1 & 4 & 24 & {$[(\mathrm{RS}-1) / 3] \times 100$} & $0.374^{*}$ \\
\hline Bleeding Gingiva & $B G$ & 25 & $1 \sim 4$ & 1 & 4 & 25 & {$[(\mathrm{RS}-1) / 3] \times 100$} & $0.997^{* *}$ \\
\hline Weight loss & $W L$ & 27 & $1 \sim 2$ & 1 & 2 & 27 & $(\mathrm{RS}-1) \times 100$ & $0.866^{* *}$ \\
\hline Weight gain & WG & 28 & $1 \sim 2$ & 1 & 2 & 28 & $($ RS-1) $\times 100$ & $/^{a}$ \\
\hline
\end{tabular}

${ }^{*} P<0.05{ }^{* *} p<0.01$

${ }^{a}$ Each subject got 0 points in the sexuality scale and weight gain scale in the retest trial (constant sequence), so these scales were not part of the calculation of the correlation coefficient

scale, was seen as a single item. Weight loss and weight gain are mutually exclusive and opposite, so they were not placed into the same scale. Factor loadings of pain in throat and diet change were high in more than one factors while that of shoulder and neck function and bleeding gingiva were relatively low with all the factors. But these items were not deleted because results may be affected by a small sample size. Nevertheless, the factor analysis demonstrated the statistical significance and clinical value of the items and scale division of the QOL-OC.

The results show that internal consistency reliability was achieved in this research. The reliability was slightly lower in the pain and discomfort and eating and saliva scales, due to statistical correlation and coherence in clinical function. Test-retest reliability for shoulder and neck function was a bit poor; this may be explained by the small size of the sample [38].

Table 4 Correlation coefficient between each scale of QOL-OC

\begin{tabular}{lllll}
\hline Scales & PD & ET & SA & SC \\
\hline PD & & & & \\
ET & $.341^{* *}$ & & \\
SA & $.232^{* *}$ & $.336^{* *}$ & $.283^{* *}$ & $.470^{* *}$ \\
SC & $.154^{*}$ & $.539^{* *}$ & $.330^{* *}$ & \\
DT & $.339^{* *}$ & $.476^{*}$ & \\
\hline Abbreviations: $P D$ pain and discomfort, ET eating, SA saliva, SC social contact, \\
$D T$ diet \\
${ }^{*} p<0.05$ & & & \\
${ }^{* *} p<0.01$ & & &
\end{tabular}

Favourable discriminant validity was indicated in that the correlation coefficient was insignificant between scales. This suggests that the different scales measure significantly different symptoms. Convergent validity was demonstrated in that the majority of the items show higher correlation coefficients with their own scales than with the other scales - greater than 0.4 in all cases but one. The painkiller item had a lower correlation (0.378), but this value was still greater than the correlation between the painkiller items and any other scales whether by horizontal or vertical comparison.

It is reported that baseline dysphagia affects multiple domains of QOL and general health perceptions in patients with head and neck cancer prior to treatment. Lango et al. suggested that a dysphagia measure captures the effort of maintaining nutrition, and identifies patients predisposed to disease recurrence and diseaserelated death [47-49]. Therefore, in our study, according to Chinese people's eating habits, the eating fluid item (Item 5) was placed within the eating scale in sequence with Items 6, 7, and 8; this decision was made because it is one item in a specific spectrum of eating-related issues which renders its inclusion necessary to achieving a logical outcome. We submit that the eating solid item may be more closely related to masticatory function while the eating fluid or semifluid items may be affected by food leakage arising from defects in the resection of the mandible or maxilla. The pain and social problems scales are present and highly correlated in both the C30 
Table 5 Spearman correlation coefficient between items and its corresponding scales of QOL-OC

\begin{tabular}{|c|c|c|c|c|c|c|}
\hline \multirow[t]{2}{*}{ Items } & & \multicolumn{5}{|l|}{ Scales } \\
\hline & & PD & ET & SA & SC & DT \\
\hline 31 & Pain in the mouth & $.694^{* *}$ & $.277^{* *}$ & $.182^{* *}$ & .050 & $.253^{* *}$ \\
\hline 32 & Pain in the jaw & $.525^{* *}$ & $.263^{* *}$ & $.245^{* *}$ & .116 & $.234^{* *}$ \\
\hline 33 & Pain in the throat & $.478^{* *}$ & $200^{* *}$ & $.199^{* *}$ & $.218^{* *}$ & $.201^{* *}$ \\
\hline 34 & Discomfort in the mouth & $.729^{* *}$ & $.324^{* *}$ & $.150^{*}$ & $.187^{* *}$ & $.315^{* *}$ \\
\hline 56 & Use of painkillers & $.376^{* *}$ & .128 & $.175^{* *}$ & .116 & $.136^{*}$ \\
\hline 35 & Eating fluid & .001 & $.372^{* *}$ & .063 & $.203^{* *}$ & .116 \\
\hline 36 & Eating semifluid & $.245^{* *}$ & $.506^{* *}$ & $.171^{*}$ & $.299^{* *}$ & $.218^{* *}$ \\
\hline 37 & Eating solid & $.322^{* *}$ & $.905^{* *}$ & $.274^{* *}$ & $.502^{* *}$ & $.451^{* *}$ \\
\hline 38 & Choked when eating & $.271^{* *}$ & $.553^{* *}$ & $.346^{* *}$ & $.325^{* *}$ & $.342^{* *}$ \\
\hline 41 & Dry mouth & $.164^{*}$ & $.211^{* *}$ & $.861^{* *}$ & $.200^{* *}$ & $.268^{* *}$ \\
\hline 42 & Sticky saliva & $.256^{* *}$ & $.341^{* *}$ & $.779^{* *}$ & $.274^{* *}$ & $.282^{* *}$ \\
\hline 45 & Difficult chatting & .043 & $.392^{* *}$ & $.183^{* *}$ & $.809^{* *}$ & $.364^{* *}$ \\
\hline 46 & Social contact with families & .113 & $.384^{* *}$ & $.193^{* *}$ & $.691 * *$ & $.241^{* *}$ \\
\hline 47 & Social contact with friends & $.136^{*}$ & $.422^{* *}$ & $.229^{* *}$ & $.789^{* *}$ & $.329^{* *}$ \\
\hline 48 & Social contact in public & $.175^{* *}$ & $.457^{* *}$ & $.183^{* *}$ & $.826^{* *}$ & $.367^{* *}$ \\
\hline 53 & Pronunciation & $.145^{*}$ & $.551^{* *}$ & $.258^{* *}$ & $.850^{* *}$ & $.457^{* *}$ \\
\hline 51 & Not enjoying eating & $.300^{* *}$ & $.453^{* *}$ & $.334^{* *}$ & $.382^{* *}$ & $.854 * *$ \\
\hline 52 & Diet change & $.283^{* *}$ & $.381^{* *}$ & $.257^{* *}$ & $.442^{* *}$ & $.905^{* *}$ \\
\hline
\end{tabular}

Abbreviations: $P D$ pain and discomfort, ET eating, SA saliva, SC social contact, DT diet Correlation coefficients between items and its corresponding scales were made in bold ${ }^{*} p<0.05$

${ }^{* *} p<0.01$

and OC. This indicates that the $\mathrm{OC}$ is coherent with a universally recognized measure for evaluation of symptoms. In addition, the OC covers more specific and distinct problems concerning pain in the oral and maxillofacial region and social problems; these are all graded at several levels.

There are also several limitations in our study. First, apart from questions about sexuality, a number of questions in eating scales and this study cannot be answered by nasogastric feeding tube users. To address this, investigators gave answers to these questions based on the patients' responses. Second, because patients with serious speaking difficulties and patients who spoke more opaque dialects were difficult to understand in the telephonic interviews, these participants were excluded by necessity. Telephonic interview may lead to selection bias, resulting from patients whose language capacity were impaired severely unable to complete the test, which is much more common among advanced oral cancer patients received surgery plus chemotherapy or/ and radiotherapy treatment. In addition to an increase of the sample amount, making investigations in multiple methods and raising the ratio of face to face interview will reduce this bias and expand the sample size of test-

Table 6 Correlation between scales of C30 and QOL-OC

\begin{tabular}{|c|c|c|c|c|c|c|c|c|c|}
\hline Scales & $\mathrm{QL}$ & PF & RF & $\mathrm{EF}$ & CF & SF & FA & NV & PA \\
\hline$\overline{P D}$ & $-.255^{* *}$ & $-.192^{* *}$ & $-.231^{* *}$ & $-.239^{* *}$ & $-.271^{* *}$ & $-.165^{*}$ & $.369^{* *}$ & $.198^{* *}$ & $.543^{* *}$ \\
\hline ET & $-.420^{* * *}$ & $-.327^{* *}$ & $-.283^{* *}$ & $-.190^{* *}$ & -.069 & $-.388^{* *}$ & $.360^{* *}$ & $.140^{*}$ & $.241^{* *}$ \\
\hline SA & $-.377^{* *}$ & $-.182^{* *}$ & $-.204^{* *}$ & $-.236^{* *}$ & $-.214^{* *}$ & $-.322^{* *}$ & $.244^{* *}$ & .087 & $.202^{* *}$ \\
\hline SC & $-.387^{* *}$ & $-.277^{* *}$ & $-.301^{* *}$ & $-.210^{* *}$ & .023 & $-.525^{* *}$ & $.278^{* *}$ & .049 & .079 \\
\hline DT & $-.359^{* *}$ & $-.237^{* *}$ & $-.344^{* *}$ & $-.220^{* *}$ & $-.172^{*}$ & $-.450^{* *}$ & $.323^{* *}$ & .103 & $293^{* *}$ \\
\hline
\end{tabular}

Abbreviations in QOL-OC: $P D$ pain and discomfort, ET eating, SA saliva, SC social contact, DT diet

Abbreviations in C30: QL global health status, $P F$ physical function, $R F$ role function, $E F$ emotional function, CF cognitive function, $S F$ social function, $F A$ fatigue, $N V$ nausea and vomiting, $P A$ pain

Data in bold referred to moderate or higher correlation

${ }^{*} p<0.05$

${ }^{* *} p<0.01$ 
retest. Third, a lack of investigators lengthened the time window for data collection. Finally, it is possible that patients might have misunderstood the timeframe for some of the questions. For example, the questions about diet change and weight change were meant to reference only the week previous to the interview, but patients tended to describe a long-term change after being sick or receiving treatment.

Although it is in need of further modification and improvement, this questionnaire is sufficiently reliable and valid for evaluating the quality of life of Chinese oral cancer patients. It is a successful preliminary step in the development of quality-of-life measures specific to Chinese patients with oral cancer.

\section{Conclusion}

Based on literature review and clinic evaluation, Oral Cancer Quality-of-life Questionnaire (QOL-OC) were designed and demonstrates fairly good statistical reliability, validity, and feasibility.

\section{Additional files}

Additional file 1: The English version of QOL-OC, this Oral Cancer Quality-of-life Questionnaire were initially designed and used for Chinese Oral Cancer patients, this is an English translation version. (PDF $298 \mathrm{~kb}$ )

Additional file 2: Scores of EORTC-C30, this table shows the primary score of patients answering the (30 questionnaire. (PDF $1500 \mathrm{~kb}$ )

Additional file 3: Scores of $\mathrm{QOL}-\mathrm{OC}$, this table shows the primary score of patients answering the C30 questionnaire include retest. (PDF $1558 \mathrm{~kb}$ )

\section{Abbreviations}

CF: Cognitive function; DT: Diet; EF: Emotional function; EORTC QLQC30: Quality of life core questionnaire 30 of the European Organisation for Research and Treatment of Cancer; EORTC: European Organization for Research and Treatment of Cancer; ET: Eating; FA: Fatigue; FACT: Functional Assessment of Cancer Therapy; FLIC: Functional Living Index-Cancer; NV: Nausea and vomiting; PA: Pain; PD: Pain and discomfort; PF: Physical function; QL: Global health status; QOL-OC: Oral Cancer Quality-of-life Questionnaire; RF: Role function; SA: Saliva; SC: Social contact; SD: Standard deviation; SF: Social function; UW-QOL: University of Washington Head and Neck Measure

\section{Acknowledgements}

We gratefully acknowledge the patients for participation in this study. We thank the Wuhan University School of Stomatology, the Wuhan University Zhongnan Hospital, Wuhan Hospice Center, Li Kashing Foundation, the Changzhou Stomatology Hospital, and Shenzhen No. 2 People's Hospital for their supports.

\section{Funding}

The work was supported by the grant 81101221 from the National Natural Science Foundation of China and grants S2014862 and S2015877 from Wuhan University. S2014862 and S2015877 covered the cost of collecting data. Expenses on analysing the data and polishing the manuscript were paid by 81101221. The funders had no role in study design and decision to publish.

\section{Availability of data and materials}

All data generated or analysed during this study are included in this published article and its supplementary information files.

\section{Authors' contributions}

PYC, JCX, LBR, YXJ, LC and NM carried out data collection and drafted the manuscript, PYC, WXY and ZSN performed the statistical analysis. NM designed, coordinated, and supervised the study and critically reviewed the manuscript. NM and LC did the follow-up. All authors have read and approved the final version of the manuscript.

\section{Ethics approval and consent to participate}

This studies have been approved by the Medical Ethics Committee of Wuhan University and the number is 2013-043. All procedures performed in studies involving human participants were in accordance with the ethical standards of the institutional and/or national research committee and with the 1964 Helsinki declaration and its later amendments or comparable ethical standards. Oral or written informed consent was obtained from each patient before participation in the study.

\section{Competing interests}

The authors declare that they have no competing interests.

\section{Publisher's Note}

Springer Nature remains neutral with regard to jurisdictional claims in published maps and institutional affiliations.

Received: 31 March 2017 Accepted: 16 April 2018

Published online: 03 May 2018

References

1. Zhang ZY. Oral and maxillofacial surgery. The seventh edition. Chap. 8. Beijing: People's Health Publishing House; 2012. p. 280-341.

2. Petersen PE. Oral cancer prevention and control-the approach of the World Health Organization. Oral Oncol. 2009;45:454-60.

3. Argiris A, Karamouzis MV, Raben D, Ferris RL. Head and neck cancer. Lancet. 2008:371:1695-709.

4. Fingeret MC, Teo I, Goettsch K. Body image: a critical psychosocial issue for patients with head and neck cancer. Curr Oncol Rep. 2015;17:422.

5. National Cancer Institute. Oral cavity and pharynx Cancer [EB/OL]. 2013; http://seer.Cancer.gov/statfacts/html/oralcav.html.

6. World Health Organization. 2014. Fact sheet no. 297: cancer [DB/MT]. WHO Media Centre.

7. Li W, Xu Z, Liu F, Huang S, Dai W, Sun C. Vascularized free forearm flap versus free anterolateral thigh perforator flaps for reconstruction in patients with head and neck cancer: assessment of quality of life. Head Neck. 2013;35:1808-13.

8. Gliklich RE, Goldsmith TA, Funk GF. Are head and neck specific quality of life measures necessary? Head Neck. 1997:19:474-80.

9. Quinten C, Martinell F, Coens C, Sprangers MA, Ringash J, Gotay C, Bjordal K, Greimel E, Reeve B, Maringwa J, Ediebah DE, Zikos E, King MT, Osoba D, Taphoorn MJ, Flechtner H, Schmucker-Von Koch J, Weis J, Bottomley A, Patient Reported Outcomes and Behavioral Evidence (PROBE) and the European Organization for Research and Treatment of Cancer (EORTC) Clinical Groups. A global analysis of multitrial data investigating quality of life and symptoms as prognostic factors for survival in different tumor sites. Cancer. 2014;120:302-11.

10. Barrios R, Montero J, González-Moles MA, Baca P, Bravo M. Levels of scientific evidence of the quality of life in patients treated for oral cancer. Med Oral Patol Oral Cir Bucal. 2013;18:e578-84.

11. Evans N, Meñaca A, Koffman J, Harding R, Higginson IJ, Pool R, Gysels M, PRISMA. Cultural competence in end-of-life care: terms, definitions, and conceptual models from the British literature. J Palliat Med. 2012;15:812-20.

12. Albers G, Hardin R, Pasman HR, Onwuteaka-Philipsen BD, Hall S, Toscani F Ribbe MW, Deliens L. What is the methodological rigour of palliative care research in long-term care facilities in Europe? A systematic review. Palliat Med. 2012;26:722-33.

13. Bjordal K, Hammerlid E, Ahlner-Elmqvist M, de Graeff A, Boysen M, Evensen JF, Biörklund A, de Leeuw JR, Fayers PM, Jannert M, Westin T, Kaasa S. Quality of life in head and neck cancer patients: validation of the European Organization for Research and Treatment of Cancer quality of life questionnaire-H\&N35. J Clin Oncol. 1999;17:1008-19.

14. Aaronson NK, Ahmedzai S, Bergman B, Bullinger M, Cull A, Duez NJ, Filiberti A, Flechtner H, Fleishman SB, de Haes JC. The European Organization for Research and Treatment of Cancer QLQ-C30: a quality-of-life instrument for use in international clinical trials in oncology. J Natl Cancer Inst. 1993;85:365-76. 
15. Cella DF, Tulsky DS, Gray G, Sarafian B, Linn E, Bonomi A, Silberman M, Yellen SB, Winicour P, Brannon J. The functional assessment of Cancer therapy scale: development and validation of the general measure. J Clin Oncol. 1993;11:570-9.

16. Schipper $\mathrm{H}$, Clinch J, McMurray A, Levitt M. Measuring the quality of life of cancer patients: the functional living index-Cancer: development and validation. J Clin Oncol. 1984;2:472-83.

17. Ware JE. SF 36 health survey manual and interpretation guide. Boston: New England Medical Center; 1993.

18. Ojo B, Genden EM, Teng MS, Milbury K, Misiukiewicz KJ, Badr H. A systematic review of head and neck cancer quality of life assessment instruments. Oral Oncol. 2012;48:923-37.

19. Singer S, Arraras JI, Chie WC, Fisher SE, Galalae R, Hammerlid E, NicolatouGalitis O, Schmalz C, Verdonck-de Leeuw I, Gamper E, Keszte J, Hofmeister D. Performance of the EORTC questionnaire for the assessment of quality of life in head and neck cancer patients EORTC QLQ-H\&N35: a methodological review. Qual Life Res. 2013;22:1927-41.

20. Infante-Cossio P, Torres-Carranza E, Cayuela A, Gutierrez-Perez JL, Gili-Miner M. Quality of life in patients with oral and oropharyngeal cancer. Int J Oral Maxillofac Surg. 2009;38(3):250-5.

21. Metcalfe CW, Lowe D, Rogers SN. What patients consider important: temporal variations by earlyand late stage oral, oropharyngeal and laryngeal subsites. J Craniomaxillofac Surg. 2014;42:641-7.

22. Sherman AC, Simonton S. Advances in quality of life research among head and neck Cancer patients. Curr Oncol Rep. 2010;12(3):208-15.

23. Moore KA, Ford PJ, Farah CS. Support needs and quality of life in oral cancer: a systematic review. Int J Dent Hyg. 2014;12(1):36-47.

24. Barrios R, Tsakos G, García-Medina B, Martínez-Lara I, Bravo M. Oral healthrelated quality of life and malnutrition in patients treated for oral cancer. Support Care Cancer. 2014;22(11):2927-33.

25. Babin E, Sigston E, Hitier M, Dehesdin D, Marie JP, Choussy O. Quality of life in head and neck cancers patients: predictive factors, functional and psychosocial outcome. Eur Arch Otorhinolaryngol. 2008;265(3):265-70.

26. Chandu A, Smith AC, Rogers SN. Health-related quality of life in oral cancer: a review. J Oral Maxillofac Surg. 2006;64(3):495-502.

27. Rathod S, Gupta T, Ghosh-Laskar S, Murthy V, Budrukkar A, Agarwal J. Quality-of-life (QOL) outcomes in patients with head and neck squamous cell carcinoma (HNSCC) treated with intensity-modulated radiation therapy (IMRT) compared to three-dimensional conformal radiotherapy (3D-CRT): evidence from a prospective randomized study. Oral Oncol. 2013:49:634-42.

28. Bjordal K, de Graeff A, Fayers PM, Hammerlid E, van Pottelsberghe C, Curran D, Ahlner-Elmqvist M, Maher EJ, Meyza JW, Brédart A, Söderholm AL, Arraras JJ, Feine JS, Abendstein H, Morton RP, Pignon T, Huguenin P, Bottomly A, Kaasa S. A 12 country field study of the EORTC QLQ-C30 (version 3.0) and the head and neck cancer specific module (EORTC QLQ-H\&N35) in head and neck patients. EORTC quality of life group. Eur J Cancer. 2000;36(14):1796-807.

29. Bottomley A, Tridello G, Coens C, Rolland F, Tesselaar ME, Leemans CR, Hupperets P, Licitra L, Vermorken JB, Van Den Weyngaert D, Truc G, Barillot I, Lefebvre JL. An international phase 3 trial in head and neck cancer: quality of life and symptom results. Cancer. 2014;120(3):390-8.

30. Wan C, Meng Q, Yang Z, Tu X, Feng C, Tang X, Zhang C. Validation of the simplified Chinese version of EORTC QLQ-C30 from the measurements of five types of inpatients with cancer. Ann Oncol. 2008;19:2053-60.

31. Sprangers MA, Cull A, Groenvold M, Bjordal K, Blazeby J, Aaronson NK. The European Organization for Research and Treatment of Cancer approach to developing questionnaire modules: an update and overview. EORTC quality of life study group. Qual Life Res. 1998;7:291-300.

32. Sprangers MA, Cull A, Bjordal K, Groenvold M, Aaronson NK. The European Organization for Research and Treatment of Cancer. Approach to quality of life assessment: guidelines for developing questionnaire modules. EORTC study group on quality of life. Qual Life Res. 1993;2:287-95.

33. Gralla RJ, Hollen PJ. Quality-of-life assessment: the challenge of incorporating quality-of-life and patient-reported outcomes into investigative trials and clinical practice. The MASCC textbook of Cancer supportive care and survivorship; 2011. p. 63-70.

34. Patrick DL, Burke LB, Gwaltney CJ, Leidy NK, Martin ML, Molsen E, Ring L. Content validity_establishing and reporting the evidence in newly developed patient-reported outcomes (PRO) instruments for medical product evaluation: ISPOR PRO good research practices task force report: part 1-eliciting concepts for a new PRO instrument. Value Health. 2011:14:967-77.
35. Hjermstad MJ, Bergenmar M, Fisher SE, Montel S, Nicolatou-Galitis O, RaberDurlacher J, Singer S, Verdonck-de Leeuw I, Weis J, Yarom N, Herlofson BB. The EORTC QLQ-OH17: a supplementary module to the EORTC QLQ-C30 for assessment of oral health and quality of life in cancer patients. Eur J Cancer. 2012;48(14):2203-11.

36. List MA, D'Antonio LL, Cella DF, Siston A, Mumby P, Haraf D, Vokes E. The performance status scale for head and neck Cancer patients and the functional assessment of Cancer therapy-head and neck scale. A study of utility and validity. Cancer. 1996;77(11):2294-301.

37. Rogers SN, Laher S, Overend L, Lowe D. Domain importance-rating using the UW-QOL questionnaire in patients treated by primary surgery for oral and oropharyngeal cancer. J Cranio Maxillofac Surg. 2002;30:125-32.

38. Wang HL, Keck JF, Weaver MT, Mikesky A, Bunnell K, Buelow JM, Rawl SM. Shoulder pain, functional status, and health-related quality of life after head and neck cancer surgery. Rehabil Res Pract. 2013;2013:601768.

39. Rogers SN, Lowe D. The University of Washington Quality of Life Scale[M]. New York: Springer; 2010.

40. Aplak B, Malko CM, Gelecek N, Sen M. Quality of life of Turkish patients with head and neck cancer. Turkish J Cancer. 2007;37:129-35.

41. Jayasekara H, Rajapaksa LC, Aaronson NK. Health-related quality-of-life in patients with head-and-neck cancer in Sri Lanka: psychometric properties of the 'Sinhala' version of the EORTC QLQ-H\&N35[J]. Psycho-Oncology. 2010; 18(10):1116-21.

42. Low C, Fullarton M, Parkinson E, O'Brien K, Jackson SR, Lowe D, Rogers SN. Issues of intimacy and sexual dysfunction following major head and neck cancer treatment. Oral Oncol. 2009:45(10):898-903.

43. Hayton JC, Allen DG, Scarpello V. Factor retention decisions in explotary factor analysis: a tutorial on parallel analysis. Organ Res Methods. 2004;7:191-205.

44. D'cruz A, Lin T, Anand AK, Atmakusuma D, Calaguas MJ, Chitapanarux I, Cho BC, Goh BC, Guo Y, Hsieh WS, Hu C, Kwong D, Lin JC, Lou PJ, Lu T, Prabhash K, Sriuranpong V, Tang P, Vu W, Wahid I, Ang KK, Chan AT. Consensus recommendations for management of head and neck cancer in Asian countries: a review of international guidelines. Oral Oncol. 2013;49(9):872-7.

45. Mcmillan SC, Weitzner M. Quality of life in cancer patients: use of a revised hospice index. Cancer Pract. 1998;6(5):282.

46. Deng D, Fu L, Zhao YX, Wu X, Zhang G, Liang C, Xie CH, Zhou YF. The relationship between cancer pain and quality of life in patients newly admitted to Wuhan hospice Center of China. Am J Hosp Palliat Care. 2012; 29(1):53-9.

47. Lango MN, Egleston B, Fang C, Burtness B, Galloway T, Liu J, Mehra R, Ebersole B, Moran K, Ridge JA. Baseline health perceptions, dysphagia, and survival in patients with head and neck cancer. Cancer. 2014;120(6):840-7.

48. Fang QG, Shi S, Zhang X, Li ZN, Liu FY, Sun CF. Assessment of the quality of life of patients with oral cancer after pectoralis major myocutaneous flap reconstruction with a focus on speech. J Oral Maxillofac Surg. 2013;71(11): 2004. e1-2004.e5

49. Djan R, Penington A. A systematic review of questionnaires to measure the impact of appearance on quality of life for head and neck cancer patients. J Plast Reconstr Aesthet Surg. 2013;66(5):647-59.

\section{Ready to submit your research? Choose BMC and benefit from:}

- fast, convenient online submission

- thorough peer review by experienced researchers in your field

- rapid publication on acceptance

- support for research data, including large and complex data types

- gold Open Access which fosters wider collaboration and increased citations

- maximum visibility for your research: over $100 \mathrm{M}$ website views per year

At BMC, research is always in progress.

Learn more biomedcentral.com/submissions 